\title{
Prevalence and Characteristics of Game Transfer Phenomena: A Descriptive Survey Study
}

\section{Angelica Beatriz Ortiz de Gortari \& Mark D. Griffiths}

To cite this article: Angelica Beatriz Ortiz de Gortari \& Mark D. Griffiths (2016): Prevalence and Characteristics of Game Transfer Phenomena: A Descriptive Survey Study, International Journal of Human-Computer Interaction

To link to this article: http://dx.doi.org/10.1080/10447318.2016.1164430

Accepted author version posted online: 16

Mar 2016.

Submit your article to this journal ¿

Q View related articles $₫$

View Crossmark data \lceil 


\title{
Prevalence and Characteristics of Game Transfer Phenomena: A Descriptive Survey Study
}

\author{
Angelica Beatriz Ortiz de Gortari \\ Email: angelica@gametransferphenomena.com \\ Psychology, Nottingham Trent University, Burton Street, Nottingham, UK, NG1 4BU
}

\section{Mark D. Griffiths}

Nottingham Trent University

Running title: Prevalence and characteristics of Game Transfer Phenomena

\begin{abstract}
\end{abstract}
Previous qualitative studies suggest that gamers experience Game Transfer Phenomena (GTP), a variety of non-volitional phenomena related to playing videogames including thoughts, urges, images, sounds when not playing. To investigate (i) which types of GTP were more common and (ii) their general characteristics, the present study surveyed a total of 2,362 gamers via an online survey. The majority of the participants were male, students, aged between 18 and 27 years, and 'hard-core' gamers. Most participants reported having experienced at least one type of GTP at some point (96.6\%), the majority having experienced GTP more than once with many reporting 6 to 10 different types of GTP. Results demonstrated that videogame players experienced (i) altered visual perceptions (ii) altered auditory perceptions (iii) altered body perceptions (iv) automated mental processes, and (v) behaviors. In most cases, GTP could not be explained by being under the influence of a psychoactive substance. The GTP experiences were usually shortlived, tended to occur after videogame playing rather than during play, occurred recurrently, and usually occurred while doing day-to-day activities. One in five gamers had experienced some 
type of distress or dysfunction due to GTP. Many experienced GTP as pleasant and some wanted GTP to happen again.

Keywords: Game Transfer Phenomena, non-volitional phenomena, Tetris Effect, priming, videogame effects

\section{INTRODUCTION}

There has been increased research investigating the psychosocial effects of playing videogames, mainly focusing on violent games (Elson \& Ferguson, 2014) and gaming addiction (Kuss \& Griffiths, 2012). However, there is still little understanding about how videogame playing influences gamers' perceptions, cognitions, and behaviors in day-to-day contexts. Consequently, the present study examines Game Transfer Phenomena (GTP) (i.e., transfer of experiences from the virtual to the physical world).

\section{Game Transfer Phenomena and related studies}

Only a few studies have examined how videogame experiences are directly transferred to reallife contexts. The term “Game Transfer Phenomena” (GTP) was for first time used in Ortiz de Gortari (2010) for described altered sensorial perceptions, mental process and behaviors transfer from the videogame world to real life context. Research into GTP has taken a multimodal research approach focusing on understanding a large variety of non-volitional phenomena with videogame contents that range from everyday normal experiences to dysfunctional intrusions. Transfer of game experiences have been examined by investigating the relationship between the 
structural characteristics of videogames (e.g., visual or aural features) and in-game activities directly related to gamers' transfer of experiences that typically manifest after stopping playing and include (i) altered sensorial perceptions, (ii) mental processes, and (iii) behaviors and actions. In many cases, GTP are triggered by game-related cues in physical contexts. The GTP approach (Ortiz de Gortari \& Griffiths, 2015) distinguishes between phenomena that manifest as: (i) inner or outer phenomena (e.g., visualizing images from the videogame in the mind, seeing images from videogames super-imposed on physical external objects in real life), (ii) selfgenerated or non-self-generated (e.g. hearing one's own thoughts, hearing voices of someone else), and (iii) voluntary or involuntary (e.g., using videogame slang for amusement or involuntary verbal outbursts using game content). The main modalities of GTP comprise the following:

Altered perceptions include perceptions and/or sensations related to the videogame when not playing and can occur across all sensory modalities. The most commonly reported have been in relation to visuals effects in the game (e.g., mind visualizations, after-images, visual hallucinations), auditory cues (e.g., auditory imagery, musical hallucinations), and kinesthetic and chronoceptive perceptions influenced by the game (Ortiz de Gortari, Aronsson, \& Griffiths, 2011; Ortiz de Gortari \& Griffiths, 2014a; 2014b).

Automatic mental processes include thoughts, urges, and automatic mental actions. These include thoughts about the game (e.g., intrusive thoughts), cognitive biases (e.g., attention bias toward videogame-related cues), and source monitoring errors (e.g., confusion of memories from 
the videogame with those in real life) (Ortiz de Gortari, et al., 2011; Ortiz de Gortari \& Griffiths, 2014c).

Behaviors and actions include actions initiated with premeditation or those experienced as involuntary. These range from involuntary motor activations (e.g. involuntary movements of fingers) to performing actions inspired by the videogame or changes in behavior related with the videogame (e.g., avoiding specific places that relate to those found in the videogame, mimicking videogame characters, having verbal outbursts using videogame narrative) (Ortiz de Gortari, et al., 2011; Ortiz de Gortari \& Griffiths, 2014c).

In the first published study examining GTP, 42 Swedish frequent gamers aged 15 to 21 years were interviewed. The four main categories identified based on thematic analysis were (i) dreams, (ii) automatic thoughts and urges (i.e., thinking about the game in day-to-day contexts), (iii) altered perceptions (i.e., visual or aural information, and body sensations), and (iv) behaviors (e.g. using narrative slang from the videogame for amusement). Gamers reported both voluntary and involuntary experiences, and the core of transfer of game experiences identified were reported as being triggered by game-related cues.

Following this first published study, a series of qualitative studies by Ortiz de Gortari and Griffiths extended the findings in the first GTP study and examined over 1,600 gamers' selfreports about GTP in different modalities (e.g., altered sensorial perceptions, mental processes and behaviors) and demonstrated that such phenomena were relatively commonplace among gamers (Ortiz de Gortari \& Griffiths, 2014a; 2014b; 2014c; Ortiz de Gortari \& Griffiths, 2015). 
To date, studies examining GTP have reported a wide variety of non-volitional phenomena related to playing videogames (e.g., seeing videogame images in the back of the eyelids, hearing music, noises and/or voices from the videogame). Also, gamers have suddenly confused physical objects and sounds with something from the videogame and have perceived physical objects and environments as distorted. For instance, gamers have reported seeing icons above people's heads or menus in the corner of their eyes (Ortiz de Gortari, et al., 2011; Ortiz de Gortari, Aronsson, \& Griffiths, 2013; Ortiz de Gortari \& Griffiths, 2014a). Other altered perceptions include feeling the body stiffen, feeling that time has slowed down, experiencing uncoordinated body movements, and/or moving automatically as they would do in the videogame (Ortiz de Gortari \& Griffiths, 2014c). Furthermore, a variety of automatic mental processes derived from engaging in gaming have been identified. These include players that keep thinking about the videogame or interpreting real-life events with the logic of the videogame. In some cases, this has resulted in automatic behaviors (e.g., approaching physical objects with the intention of doing something as in the videogame and then realizing they were not in the game any longer, suddenly mimicking movements of videogame characters, verbal outbursts utilizing game content, etc.) (Ortiz de Gortari, et al., 2011; Ortiz de Gortari \& Griffiths, 2014c).

A few other studies have examined transfer effects when playing videogames (e.g., Bigl, 2013; Fritz, 2005; Poels, Ijsselsteijn \& de Kort, (2014). Fritz (2005) identified ten transfer types based on qualitative research (i.e., problem-solving transfer, emotional transfer, instrumental actionoriented transfer, ethical-moral transfer, associative transfer, reality-structuring transfer, informational transfer, memory-related transfer, time-experiencing transfer, and transfer related to imaginative activity). Bigl (2013) conducted an online survey $(\mathrm{n}=1,146)$ and found that $87 \%$ 
of the participants reported transfer effects, based on the ones described by Fritz (2005). The main similarities between the GTP research of Ortiz de Gortari and Griffiths, and those of Fritz and Bigl is that they all make inference to automatic associations between elements in the game and those in real life context. However, Fritz's (2005) perspective focuses on the transfer of learned skills, emotions, morals, and ethics from the game to the real world (e.g., problem solving, analysis ability) rather than sensory transfer.

Poels, Ijsselsteijn and de Kort's (2014) carried out a factorial analysis of an online survey with 511 World of Warcraft gamers examining (i) memory recalls from the game triggered by stimuli found in real life contexts (physical objects, sounds and music), (ii) game biases perceptions (e.g. cravings for playing), and (iii) inclusion of game content in daydreams, nightly dreams, vocabulary and expressions. There are large similarities and overlaps between this study and findings in the first study published concerning GTP (Ortiz de Gortari, et al., 2011) including the use of videogame slang, and dreams/thoughts about the videogame when not being playing.

Taking the small literature as a whole, some GTP related studies comprise transfer of perceptions, thoughts and behaviors (Ortiz de Gortari \& Griffiths, 2014a; 2014b; 2014c; Poels et al., 2014), while others focus on examine transfers of mental-based processes, emotions and behaviors (Fritz, 2005; Bigl, 2013), making comparisons between the studies difficult. Furthermore, even within studies examining the same types GTP process have major differences. For instance, Poels et al. (2014) only investigated gamers playing MMORPGs (Massively Multiplayer Online Role Playing Games) whereas the studies of Ortiz de Gortari and Griffiths examined all game genres, types, and medium (i.e., both online and offline games). 


\section{Characteristics of GTP}

Research studies have mainly focused on understanding which specific types of videogame content are transferred to the real world (Bigl, 2013), with little research examining the characteristics of the phenomena themselves (e.g., how long GTP last). Based on qualitative studies, the characteristics described in this section include; (i) incubation period of manifestation, (ii) circumstance of manifestation, (iii) duration of GTP, and (iv) appraisal and impact of GTP (Ortiz de Gortari, Aronsson \& Griffiths, 2011; Ortiz de Gortari \& Griffiths, 2014a; 2014b; 2014c).

Incubation period of manifestation - Gamers have reported GTP first manifesting over different time periods ranging from relatively shortly after first playing to years afterwards (Ortiz de Gortari \& Griffiths, 2014a; 2014c). The qualitative studies by Ortiz de Gortari and Griffiths (2014a; 2014b; 2014c) suggest that GTP should be considered as post-play phenomena. Knowing more about how long after playing GTP occurs can help in developing recommendations in terms of those GTP that could compromise gamers' functioning in day-today contexts. Also, knowing when GTP are more likely to be experienced may help in more accurately explaining the nature of GTP (e.g., physiological, perceptual, and cognitive mechanisms).

Circumstance of manifestation - Gamers have reported GTP when being exposed to stimuli but also to limited stimuli such as lying in bed or falling asleep or in daily life contexts particularly while engaging in automatic tasks when the mind wanders away (e.g., driving, walking, in a lecture) (Ortiz de Gortari \& Griffiths, 2014a; 2014b; 2014c). Also, gamers have reported 
experiencing GTP when feeling fatigued, stressed and/or anxious (Ortiz de Gortari, et al., 2011). Hearing or seeing images from the game intermittently while falling asleep may have no further consequences. However, seeing such images while driving or responding impulsively to stimuli associated with the game may leave individuals in potentially risky situations.

Duration of GTP - Gamers have reported that GTP can last for either short (i.e. a few seconds) or long periods of time (e.g., longer than a day). Sounds and images have been experienced for long periods of times (e.g., hours or longer), but have also been reported as being experienced episodically (Ortiz de Gortari \& Griffiths, 2014a; 2014b). While transient phenomena may have no further consequences, experiencing GTP for long periods of time may provoke distress. According to gamers' self-reports, some experienced insomnia because they kept hearing or seeing something from the games while trying to sleep. Others were annoyed when videogame images interfered with their activities (e.g., watching a movie in the cinema) (Ortiz de Gortari \& Griffiths, 2014a; 2014b).

Appraisal and impact of GTP - Different types of GTP may have different consequences according to how they are experienced, interpreted, and under what circumstances they are experienced. Therefore it is important to understand how the gamers appraise their GTP. Gamers have appraised GTP as both positive (e.g., "entertainment,” "fun,” “creative”) and negative (e.g., “sign of madness”, “scary,” “annoying,”) (Ortiz de Gortari \& Griffiths, 2014a; 2014b; 2014c).

To date, there has been little research examining how common GTP are in each modality and sub-modality. Therefore the aim of the present study was to examine the prevalence of GTP among a relatively large population of gamers. This was done with the purpose of establishing 
benchmark data so that future comparisons can be made between GTP and non-volitional phenomena reported in previous research such as the prevalence of hallucinations in the general population (Clark \& Rhyno, 2005; McGrath, Saha, Al-Hamzawi, \& et al., 2015; Ohayon, 2000). Moreover, the consequences of GTP may depend upon how long they last, when they manifest, and how they are appraised. Therefore the present study also investigates these characteristics relating to GTP. More specifically, the research questions investigated were: (i) how commonly are GTP experienced? (ii) which are the most common modalities (e.g., altered sensorial perceptions), sub-modalities (e.g., visual, auditory) and types of GTP? (iii) what are the general characteristics of GTP (e.g., duration, appraisal, videogame genre)?

\section{METHOD}

A total of 2,362 gamers completed an online survey about their GTP experiences.

Sample: Most participants were male (86.1\%), and described themselves as hard-core gamers (59.9\%). Nearly half of the participants (46.1\%) were aged between 18 and 22 years (with ages ranging from 18 to 54 years or older). Additionally, almost half of the participants (46.1\%) were students, while $28.5 \%$ were full-time employed. Participants resided in 78 different countries including Mexico (33.8\%), United States (20.0\%), United Kingdom (11.4\%), Sweden (5.4\%) and Canada (4.7\%) (see Tables 1 and 2). The response rate was high (92\%).

$\bullet$

Procedure: Participants were invited to answering an online survey, which took to answering approximately 10 minutes. They were recruited via online gaming community forums, Facebook, and meetup.com groups. Informed consent was obtained from all participants prior to 
data collection. Ethical approval was granted by the researchers' University Ethics Committee. All data were exported into SPSS 21 for analysis.

Material: Survey items in the present study were based on previously published qualitative studies into GTP (Ortiz de Gortari, 2010; Ortiz de Gortari, et al., 2011; Ortiz de Gortari, et al., 2013; Ortiz de Gortari \& Griffiths, 2014a; 2014b; 2014c). The survey comprised a total of 30 items and included questions about: socio-demographics, videogame playing habits, playing motivations, being under the influence of psychoactive substances when experiencing GTP. Three modalities of GTP were investigated; all items were scored on a Likert scale that assessed frequency (i.e., ‘all the time’, 'many times', ‘a few times', 'once' and 'never'). GTP experiences include perceptual distortions, hallucinatory-like experiences, source monitoring errors, cognitive flexibility experiences, automatic responses toward game-related cues, and change in behavior. The modalities investigated were as following:

(i) Altered perceptions: This modality was divided into sub-modalities: Altered visual (e.g., “seeing videogame images with eyes open”, “Seeing distorted real life environments and/or objects”), auditory (e.g., “hearing a sound from a videogame when not playing”, “misinterpreting a sound in real life as something from a videogame”), and body perceptions (e.g., "experiencing tactile sensations associated with a videogame”, "experiencing bodily sensations of movement as if in a videogame” etc.).

(ii) Automatic mental processes: This modality included experiences such as "wanting or feeling the urge to do something in real life after seeing something that provided reminders of the videogame”, and “momentarily mixing up videogame events with actual real life events”). 
(iii) Behaviors and actions: This modality included experiences such as "singing, shouting or saying something from a videogame in real life unintentionally, “Acting differently in real life situations because of something unintentionally experienced in a videogame” etc.).

The questionnaire also included questions regarding GTP characteristics as multiple-choice questions (e.g., circumstances, duration, emotional appraisal, impact, videogames associated, etc.). It should also be noted that the 20 items specifically relating to GTP from these data were psychometrically analyzed in another study (see: Ortiz de Gortari, Pontes, \& Griffiths, 2015). A confirmatory factor analysis (CFA) confirmed the factorial structure of the items developed and demonstrated good reliability and validity (including population cross-validity and criterionrelated validity).

Statistical analysis: As a first stage of understanding GTP as distinct phenomena, the statistical analysis in this study focused on analyzing the frequency of the GTP modalities/sub-modalities and characteristics of GTP (i.e., purely descriptive data). Only participants that responded affirmatively to at least one of the GTP items could answer the section relating to GTP characteristics. Not all participants answered every question. Therefore, sample sizes are sometimes different in the analysis of different questions. 


\section{RESULTS}

\section{Gaming habits.}

Most gamers played 2 to 4 times per week (35.2\%) or every day (34.3\%), and typically played sessions of $1-3$ hours (43.2\%). Almost the same percentage played sessions of 3-6 hours (41.3\%). Fewer participants played 1-3 hours (24.8\% when GTP occurred vs. 43.2\% typical session) or 3-6 hours (35.9\% when GTP occurred vs. 41.3 typical session) when GTP occurred. When GTP occurred, more gamers played 6 hours or more (23.0\% when GTP occurred vs. 11.5 typical session) (see Table 2).

\section{GTP frequency and GTP type.}

Almost all gamers had experienced GTP at least at some point (96.6\%), and most had experienced GTP more than once (95.3\%). In terms of the number of GTP occurrences, there was an almost equal distribution. Most gamers had experienced 6 to 10 different types of GTP (28.9\%), 11 to 15 (27.2\%), 16 to 20 (22.8\%) and fewest had experienced 1 to 5 (17.4\%).

\section{GTP modalities, sub-modalities and types.}

The modality of altered perceptions included visual, auditory, and body experiences. The other two modalities were automatic mental processes and behaviors. Large percentages were found across all modalities but the most prevalent modality/sub-modality was automatic mental processes (87.4 \%) and the least prevalent was altered body perceptions (72.7\%). The most prevalent types in each modality were: visualizing videogame elements in their mind or with 
closed eyes (76.8\%), hearing the music when not playing (73.9\%), feeling body movements after playing a game (50.9\%), thinking of using something from the videogame in real-life (74.6\%) and saying, singing or shouting something with videogame content unintentionally (57.9\%). Least prevalent in each modality were: feeling as if the mind had been disconnected from the body (28.5\%), seeing videogame images with open eyes (30.6\%), acting out behaviors or performing activities influenced by something from a videogame (39.8\%), mixing up videogame events with actual real-life events (42.5\%), and hearing voices from videogame characters (45.9\%) (see Table 3).

\section{GTP characteristics.}

The GTP characteristics were asked as multiple-choice questions as they may have varied according to each GTP. Consequently, some percentages are larger than 100\% (see Table 5). More than half had experienced GTP with videogame genres such as adventure (54.2\%) and role-playing games (53.4\%). Most participants played 11 to 20 hours per week when GTP occurred (19.8\%) while the least played 51 hours or more per week (4.8\%), and the playing session lengths when GTP occurred were for the majority either 3-6 hours (35.9\%), or 1-3 hours (24.8\%). GTP were experienced shortly after stopping playing by most, either hours after (47.0\%) or directly after stopping playing (42.4\%). The majority had experienced GTP while doing other activities excluding falling asleep or waking up (61.5\%). The duration of the GTP were mostly short, seconds (58.6\%), or minutes (29.6\%) but more than half of the players (66.2\%) had experienced GTP recurrently or episodically at some point triggered by association of stimuli or elicited by blinking. Almost half (47.2\%) reported that they had not had any special 
feelings about experiencing GTP and the majority (80.0\%) were not distressed or affected socially, occupationally, or in other areas of their day-to-day functioning due to their GTP experiences. The majority of the participants were not under the influence of any substance (medicine, alcohol or drugs) when GTP occurred (87.2\%) (see Tables 2 \& 4).

\section{DISCUSSION}

The aim of the present study was to descriptively investigate which GTP were more common and the nature of general GTP characteristics. Almost all participants reported having experienced at least one GTP at some point (96.6\%) with most reporting 6 to 10 different types of experiences. Similarly, Bigl (2013) from his computer transfer effect approach based on Fritz (2005) found that $87 \%$ of the survey participants reported transfer effects.

\section{Characteristics of GTP}

GTP were experienced in different ways, under different durations, and in a variety of circumstances. However, a number of generalities were found. GTP appear to be related with a wide variety of videogame genres. Just over a half of the gamers reported having experienced GTP after playing adventure and role-playing games, although this may also be explained by the popularity of these genres. Four hundred unique games were reported in relation to GTP (Ortiz de Gortari, 2015). For instance, seeing recurrent afterimages in the back of the eyelids were reported after playing games with stereotypical and monotonous patterns whereas automatic thoughts about the game were reported after playing games that involved repetition of activities 
such as climbing, jumping, etc. (Ortiz de Gortari, 2015). Poels et al. (2014) reported game biases and associations among MMORPG players. In most of the cases, GTP were not experienced under the influence of substances (i.e., alcohol, drugs or medicine).

Playing very long sessions to experience GTP was not necessary. More gamers played 3 to 6 hours when GTP occurred but a sizeable number played only 1 to 3 hour sessions but still experienced GTP. When comparing the typical game sessions of when GTP occurred, less gamers played 1 hour to 6 hours, while more played 6 hours or longer. In previous studies, gamers reported playing a full day or for days before GTP were experienced (Ortiz de Gortari \& Griffiths, 2014a). While some GTP may require less intensive playing to manifest, others may require longer sessions and repetitive exposure. Poels et al. (2014) found that playing frequency was positively correlated with all the domains of game transfer experiences they described (i.e., the more time gamers spent playing, the more likely they were to experience transfer experiences).

Future studies should examine the relation between playing habits with GTP modalities individually to confirm if there are differences in terms of GTP modalities.

In a previous GTP study, gamers reported experiencing sudden involuntarily thoughts, urges and body movements. In most cases, these arose as responses to physical objects or events in real life context that reminded them of something in the videogame (Ortiz de Gortari \& Griffiths, 2014c), while seeing or hearing something from the game could last longer periods of time and sometimes led to sleep deprivation (Ortiz de Gortari \& Griffiths, 2014a; 2014b). In the present 
study, GTP were usually short-lived (i.e., seconds or minutes), although more than half had experienced GTP recurrently.

Also, GTP tend to occur after playing rather than whilst playing, either immediately after playing or after hours of playing. This is important to be aware of, because confirming that gamers are more susceptible to experience GTP shortly after playing can help in developing user recommendations to reduce possible risks. However, GTP can occur when triggered by associations and do not necessarily occur shortly after playing (Ortiz de Gortari \& Griffiths, 2014a; Ortiz de Gortari \& Griffiths, 2014c). GTP appear to occur during periods where the gamers were playing intensively or were heavily engaged in the game. Future studies should investigate the incubation period before GTP manifest across different GTP modalities/submodalities. This would help to determine the potential negative or positive consequences of GTP.

Gamers have also reported experiencing GTP alone or when they were in social contexts (Ortiz de Gortari \& Griffiths, 2012; Ortiz de Gortari \& Griffiths, 2014a; 2014c). In previous studies, gamers reported GTP when doing automated activities such as driving, eating, waiting, training, etc (Ortiz de Gortari \& Griffiths, 2014a; 2014b). The findings in the present study suggest that GTP were more likely to have occurred in daytime social contexts rather than in nocturnal settings (e.g., lying in bed). While experiencing GTP alone can be uncomfortable and perhaps distressing if they occur for long periods of time (e.g., hearing music constantly, or seeing images when eyes are closed) or if they are interpreted as a sign of mental illness, experiencing GTP in social context can in extreme cases have further consequences for the player and others 
around them. The consequences of GTP depend on the circumstances of when they are experienced (e.g., day-to-day context) and if the gamer can stay in control and perform (or not perform) game-related actions when appropriate. For example, some gamers have reported trying to follow the images from the videogames they see on the road while driving (Ortiz de Gortari \& Griffiths, 2014a). Another one said he was close to driving between two cars as if in the videogame, while others have said that they avoided an accident when driving on an icy road because they responded as if they were in the game (Adde, 2011; Ortiz de Gortari \& Griffiths, 2014c).

One in five of gamers in the present study had experienced distress or had been affected in some area of their life due to their GTP experiences. In terms of appraisal of GTP, the majority of the gamers had no specific feelings, and in general, more gamers experienced GTP as something pleasurable and some wanted the GTP to occur again. Similarly, analysis of gamers self-reports revealed both positive and negative feelings concerning GTP, although more negative than positive appraisals have been reported (Ortiz de Gortari \& Griffiths, 2014a; 2014b; 2014c).

\section{Modalities and types of GTP}

Very high levels of GTP were observed among all the modalities (i.e., altered sensorial perceptions, automatic mental processes and involuntary behaviors).

\section{Altered visual perceptions}

According to previous research, nocturnal hallucinations (e.g., hypnagogic images) are likely to occurred among non-clinical populations, and even though daytime hallucinatory phenomena 
have been reported, they are strongly associated with pathology (Collerton, Perry, \& McKeith, 2005; Ohayon, 2000) - similar to the findings in the present study. The least prevalent type in the visual sub-modality was seeing videogame elements with open eyes, whereas the most common was visualizing or seeing videogame elements when eyes were closed. In previous qualitative studies concerning GTP, gamers reported that images arose recurrently, sometimes moving, and either preserved the color from the game (positive afterimages) or appeared in an opposite color (negative afterimages) (Ortiz de Gortari, et al., 2011; Ortiz de Gortari \& Griffiths, 2014a). Experimental studies have successfully induced seeing images at sleep onset (Kusse, Shaffii-Le Bourdiec, Schrouff, Matarazzo, \& Maquet, 2012; Stickgold, Malia, Maguire, Roddenberry, \& O'Connor, 2000; Wamsley, Perry, Djonlagic, Reaven, \& Stickgold, 2010). Also, visual hallucinations after exposure to a Virtual Reality system were reported in an experiment conducted with Parkinson's Disease patients, which suggests the importance in understanding individual susceptibility and distinguishing between GTP and symptoms of other underlying conditions (Albani et al., 2015).

Moreover, observations have been made of similarities between gamers' visual experiences and visual disturbances as side-effects of hallucinogen drugs (Ortiz de Gortari, 2015; Ortiz de Gortari \& Griffiths, 2014a) that manifest as "transient, recurrent, triggered-precipitate or spontaneous and visually benign” (e.g., geometric hallucinations, positive afterimages) (Lerner, Rudinski, Bor, \& Goodman, 2013 p., 297). Visual disturbances as side-effects of drugs appear to be explained by the excitation or inhibition in the visual networks related to dysfunction of serotonergic regulation (Abraham \& Duffy, 2001) and dopaminergic regulation. Dysregulation of dopaminergic transmission has been associated with hallucinations in Parkinson's Disease 
(Fénelon, Mahieux, Huon, \& Ziégler, 2000) and in schizophrenia (Abi-Dargham et al., 2000). Gamers classified as gaming addicts also show dopamine imbalance (Han et al., 2007). Therefore, even though gaming does not involve the ingestion of psychoactive substances and such alterations may be not comparable, it has been suggested that the relation between visual disturbances induced by videogame playing and disequilibrium in neurotransmission should be further investigated (Ortiz de Gortari, 2015).

Seeing images with open eyes have been reported either as (i) videogame images that manifest directly after stopping playing like typical after-images without any context, or (ii) videogame images triggered by physical objects which have been simulated in the game, or when gamers were engaged in activities that resembled activities in the game (e.g., seeing menus in conversations or power bars above peoples’ heads, etc.) (Ortiz de Gortari, et al., 2011; Ortiz de Gortari \& Griffiths, 2014a). According to cognitive theories of hallucinations (Laroi \& Woodward, 2007), these experiences may be considered misattributions of thoughts that are materialized as images (Ortiz de Gortari, 2015).

\section{Auditory altered perceptions}

The most prevalent auditory type was hearing music from the videogame when not playing. Hearing involuntary music imagery (Liikkanen, 2012) or hearing phantom mobile phone ringing (Deb, 2014) are common phenomena among the non-clinical population. The exposure to practicing and listening to music has been positively related to the frequency of the involuntary music imagery (Liikkanen, 2012) therefore hearing music from the game may be more likely to occur after playing games with background music or musical/dance games as a previous 
qualitative study showed (Ortiz de Gortari \& Griffiths, 2014b). The auditory experience that was least common was hearing voices from characters. Hearing voices appears to resemble verbal auditory hallucinations (Daalman et al., 2011), although in previous GTP studies, gamers reported hearing the voices in their head which is more closely related to inner-speech than hallucinations (Jones \& Fernyhough, 2007) but also coming from nowhere or from objects (Ortiz de Gortari \& Griffiths, 2014b). Both phenomena are usually triggered by objects or events in real-life contexts. For instance, one gamer heard commands from Team Fortress in his head, “Go go go", when he wanted people to move in the subway. Another heard a voice saying "loook" when passing by a painting (Ortiz de Gortari \& Griffiths, 2014b).

\section{Altered body perceptions}

Altered body perception was the sub-modality with lowest prevalence. The most common experience in this sub-modality was experiencing bodily sensations of movements as if they were in the videogame. This appears to be explained by the neural adaptation to the movement in the virtual environment as a post-play after-effect usually related to motion sickness. Perceiving the sensation of motion after being exposed to passive motion typically occurs after traveling on specific vehicles (e.g., boats) and is related to land sickness, post-motion vertigo, and disembarking sickness that can last up to 48 hours (Hain, Hanna, \& Rheinberger, 1999). In previous studies, gamers reported feeling their bodies moving when lying in bed after playing (Ortiz de Gortari \& Griffiths, 2014a). The lowest prevalence was reported in among those who felt as though their mind had disconnected from their body after playing (e.g., out-of-body experience). 
Virtual immersion has been associated with the disruption of multisensory integration in the vestibular, proprioceptive, tactile, and visual systems, and are considered an important precursor of disembodiment experiences such as out-of-body experiences and in autoscopy (Blanke \& Mohr, 2005). On the other hand, it could be the case that gamers experienced feelings of detachment from surroundings such as derealization and depersonalization, although this has been reported in relation to highly immersive virtual environments (Aardema, O'Connor, Côté, \& Taillon, 2010) but some individuals may be susceptible when playing videogames on conventional screens particularly due to the sophistication of contemporary videogames that are effective in inducing presence, and thus becoming comparable to virtual reality (VR) environments (Chung \& Gardner, 2012).For instance, one gamer reported that after playing a game he stood up and for a few seconds he thought he was a Jedi knight in a cave and was worried that the giant birds from the game would attack him (Ortiz de Gortari \& Griffiths, 2014c).

\section{Automatic mental processes}

The modality with most responses was automatic mental processes. Repeated use of elements in the videogame appears to become habitual and transferable to real life contexts. Wanting to use something from a videogame in real-life was the highest type reported in this modality. Sometimes these experiences manifested as spontaneous thoughts while others manifested as mishaps such as slips of actions ("when a thought that was not intended to be voiced or performed gets done anyway” (Norman, 1981 p., 3). These tend to be activated by external stimuli or internal thoughts, the activation of schema networks can take place even with just 
partial matches between stimuli (Norman, 1981). Previous studies suggest that gamers think about using videogame elements in real life contexts when their use is convenient. For instance, some gamers wanted to save real-life events and one gamer got disoriented because he could not find an arrow pointing out his destination (Ortiz de Gortari, et al., 2011; Ortiz de Gortari \& Griffiths, 2014a).

The least common experience in this modality was suddenly mixing-up videogame events with actual real-life events. Memories from virtual experiences can be very vivid. They share among other things, perceptual, spatial and emotional similarities with those memories from the real world (Johnson, 2007), therefore in certain circumstances this can lead to confusion. Previous GTP studies have found source-monitoring errors when memories from the videogame were confused with real ones (Johnson, 2006), or with reasoning biases (Jacobsen, Freeman, \& Salkovskis, 2012) when gamers found themselves expecting that something should happen in real-life as in the videogame (Ortiz de Gortari \& Griffiths, 2014a; 2014b; 2014c).

\section{Behaviors and actions}

Finally, in terms of involuntary behaviors and actions, the most prevalent was saying something using content from the videogame without intending to. Speech errors or slips of the tongue (Goldstein, Pouplier, Chen, Saltzman, \& Byrd, 2007) were also reported in a previous study and gamers have also found themselves humming music or making sounds from the videogame (Ortiz de Gortari \& Griffiths, 2014c). Acting out a behavior or performing an activity influenced by a videogame was the least common type in this modality. Interestingly, while fewer gamers performed an activity or behavior influenced by the game, at a conscious level, more gamers 
were influenced in the way they perceived and reacted to situations associated with the videogame For instance, gamers have ducked when seeing CCTV cameras, or approached objects in real-life thinking that they need to do something as they would in a videogame, then realizing they are not in the game (Ortiz de Gortari, et al., 2011; Ortiz de Gortari \& Griffiths, 2014c). Involuntary behaviors and reproduction of deliberative behaviors utilizing videogame content such as mimicking videogame characters or using slang from videogames have been reported (Ortiz de Gortari, 2010; Ortiz de Gortari, et al., 2011; Ortiz de Gortari \& Griffiths, 2014c; Poels, et al., 2014). It is important to point out that in previous studies when gamers have actually done something based on GTP, in most of the cases their actions were harmless (Ortiz de Gortari \& Griffiths, 2014c).

\section{LIMITATIONS}

The present study has a number of limitations as it was exploratory, cross-sectional, self-report, and self-selecting (and therefore subject to well known biases that may have affected data, e.g., recall bias, social desirability bias, etc.) and cannot say anything about causation. However, it is the first study to examine GTP modalities using a survey. Another limitation was that the study only some types of manifestations were addressed but GTP manifest in a large variety of ways. The GTP characteristics provide an overview in understanding GTP but it was not possible to focus on each GTP modality to keep the survey short. Future studies should examine the characteristics of GTP in each modality. Finally, the high prevalence of GTP should be interpreted with caution, as those that answered the survey may be those that recognized and had 
experienced GTP. This may explain the high prevalence of GTP. Future studies should investigate GTP in more representative samples of gamers.

\section{CONCLUSIONS AND IMPLICATIONS}

Gamers’ self-reports about GTP have been analyzed previous to this study, but it was unexpected to find such a high prevalence of GTP in the population studied. The present study highlight the pervasiveness of videogame content in gamers' lives and demonstrated that videogame playing can induce altered visual perceptions mainly manifesting as visualizations of videogame images or seeing videogame images with closed eyes; altered auditory perceptions, mainly hearing the music from the videogame; altered body perceptions mainly experiencing bodily sensations of movement as in the videogame; automatic mental processes mainly wanting to use videogame elements in real-life context; and involuntary actions when thoughts suddenly were verbalized without intention.

Supporting findings of previous studies (Ortiz de Gortari, et al., 2011; Ortiz de Gortari \& Griffiths, 2014c; Poels, et al., 2014), gamers’ experiences in the present study showed the brain’s capacity to adapt and establish associations between stimuli (brain plasticity) (Kourtzi \& DiCarlo, 2006). However, GTP experiences also showed failures of cognitive control and executive functioning when gamers were unable to override habitual sequences of responses to physical stimuli simulated in the game responding to those in similar ways as in the game, or when thoughts, urges, images, and sounds with videogame content arose while the gamers were engaged in tasks not related to the game (Ortiz de Gortari, 2015). Attention bias and disinhibition 
toward stimuli associated with gaming have been demonstrated experimentally (Metcalf \& Pammer, 2011; Van Holst et al., 2012) and studies suggest that videogame experiences contribute to failures of cognitive control (Bailey, West, \& Anderson, 2010; Kronenberger et al., 2005; Mathews et al., 2005).

It is important to point out that in most of the cases when gamers have reported reviving images or sounds when not playing, the gamers knew that the videogame images were not real, although some interacted or responded impulsively to the images (Ortiz de Gortari \& Griffiths, 2014a). Some GTP experiences can be disconcerting and worrisome as have been reported by gamers in previous studies (Ortiz de Gortari \& Griffiths, 2014a; 2014b; 2014c). Fortunately, in this study, only $20 \%$ of the gamers had experienced distress or had been affected in some area of their lives due to their experiences. Less than one-tenth of gamers had experienced GTP as unpleasant. The content, frequency, and circumstances where GTP were experienced have been suggested to be important for how GTP are appraised (e.g., Ortiz de Gortari \& Griffiths, 2014a; 2014b). The effects of priming and neural adaptations manifest in day-to-day context derived from playing

videogames should be investigated further, particularly due to the commercialization of Virtual Reality devices that enhance immersive experiences (Martínez, García, Oliver, Molina, \& González, 2014), assessing the implications of the prevalence of GTP experiences for certain periods of times.

\section{ACKNOWLEDGMENTS}

The authors are thankful to the gamers who took the time answering the survey, and the groups' moderators that promoted the survey in their venues. 


\section{AUTHORS DISCLOSURE STATEMENT}

No competing interests exist.

\section{REFERENCES}

Aardema, F., O'Connor, K., Côté, S., \& Taillon, A. (2010). Virtual Reality induces dissociation and lowers sense of presence in objective reality. CyberPsychology, Behavior and Social Networking, 13(4), 429-435.

Abi-Dargham, A., Rodenhiser, J., Printz, D., Zea-Ponce, Y., Gil, R., Kegeles, L. S., . . Laruelle, M. (2000). Increased baseline occupancy of D2 receptors by dopamine in schizophrenia. Proceedings of the National Academy of Sciences of the United States of America, 97(14), 81048109.

Abraham, H. D., \& Duffy, F. H. (2001). EEG coherence in post-LSD visual hallucinations. Psychiatry Research: Neuroimaging, 107(3), 151-163.

Adde, S. (December, 2011). Game worlds leak into reality. NewScientist. , 76-77.

Albani, G., Pedroli, E., Cipresso, P., Bulla, D., Cimolin, V., Thomas, A., . . Riva, G. (2015).

Visual Hallucinations as Incidental Negative Effects of Virtual Reality on Parkinson's Disease Patients: A Link with Neurodegeneration? Parkinson’s Disease, 2015.

Bailey, K., West, R., \& Anderson, C. A. (2010). A negative association between video game experience and proactive cognitive control. Psychophysiology, 47(1), 34-42. 
Bigl, B. (2013). If the game goes on. Perceived transfer effects from virtual game worlds into everyday life In B. Bigl \& S. Stoppe (Eds.), Playing with Virtuality, Theories and Methods of Computer Game Studies (pp. 135-146). Frankfurt am Main: Peter Lanng.

Blanke, O., \& Mohr, C. (2005). Out-of-body experience, heautoscopy, and autoscopic hallucination of neurological origin: Implications for neurocognitive mechanisms of corporeal awareness and self-consciousness. Brain Research Reviews, 50(1), 184-199.

Chung, J., \& Gardner, H. J. (2012). Temporal Presence Variation in Immersive Computer Games. International Journal of Human-Computer Interaction, 28(8), 511-529.

Clark, D. A., \& Rhyno, S. (2005). Unwanted intrusive thoughts in nonclinical individuals. In D. A. Clark (Ed.), Intrusive thoughts in clinical disorders: Theory, research, and treatment (pp. 129). New York: The Guilford Press.

Collerton, D., Perry, E., \& McKeith, I. (2005). Why people see things that are not there: A novel perception and attention deficit model for recurrent complex visual hallucinations. Behavioral and Brain Sciences, 28(6), 737-757.

Daalman, K., Boks, M., Diederen, K., de Weijer, A. D., Blom, J. D., Kahn, R. S., \& Sommer, I. (2011). The same or different? A phenomenological comparison of auditory verbal hallucinations in healthy and psychotic individuals. Journal of Clinical Psychiatry, 72(3), 320325.

Deb, A. (2014). Phantom vibration and phantom ringing among mobile phone users: A systematic review of literature. Asia-Pacific Psychiatry. 
Elson, M., \& Ferguson, C. J. (2014). Twenty-five years of research on violence in digital games and aggression. European Psychologist, 19(1), 33-46.

Fénelon, G., Mahieux, F., Huon, R., \& Ziégler, M. (2000). Hallucinations in Parkinson's disease: Prevalence, phenomenology and risk factors. Brain 123(4), 733-745.

Fritz, J. (2005). How Virtual Worlds Affect Us: On the Structure of Transfers from the Media World to the Real World. In G. Buurman (Ed.), Total Interaction (pp. 95-121): Birkhäuser Basel. Goldstein, L., Pouplier, M., Chen, L., Saltzman, E., \& Byrd, D. (2007). Dynamic action units slip in speech production errors. Cognition, 103(3), 386-412.

Hain, T. C., Hanna, P. A., \& Rheinberger, M. A. (1999). Mal de debarquement. Archives of Otolaryngology-Head and Neck Surgery, 125(6), 615-620.

Han, D. H., Lee, Y. S., Yang, K. C., Kim, E. Y., Lyoo, I. K., \& Renshaw, P. F. (2007). Dopamine genes and reward dependence in adolescents with excessive internet video game play. Journal of Addiction Medicine, 1(3), 133-138.

Jacobsen, P., Freeman, D., \& Salkovskis, P. (2012). Reasoning bias and belief conviction in obsessive-compulsive disorder and delusions: Jumping to conclusions across disorders? British Journal of Clinical Psychology, 51(1), 84-99.

Johnson, M. K. (2006). Memory and reality. American Psychologist, 61(8), 760-771.

Johnson, M. K. (2007). Reality monitoring and the media. Applied Cognitive Psychology, 21(8), 981-993. 
Jones, S. R., \& Fernyhough, C. (2007). Thought as action: Inner speech, self-monitoring, and auditory verbal hallucinations. Consciousness and cognition, 16(2), 391-399.

Kourtzi, Z., \& DiCarlo, J. J. (2006). Learning and neural plasticity in visual object recognition. Current Opinion in Neurobiology, 16(2), 152-158.

Kronenberger, W. G., Mathews, V. P., Dunn, D. W., Wang, Y., Wood, E. A., Giauque, A. L., . . . Li, T. Q. (2005). Media violence exposure and executive functioning in aggressive and control adolescents. Journal of Clinical Psychology, 61(6), 725-737.

Kuss, D. J., \& Griffiths, M. D. (2012). Online gaming addiction in children and adolescents: A review of empirical research. Journal of Behavioral Addictions, 1(1), 3-22.

Kusse, C., Shaffii-Le Bourdiec, A., Schrouff, J., Matarazzo, L., \& Maquet, P. (2012). Experience-dependent induction of hypnagogic images during daytime naps: A combined behavioural and EEG study. Journal of Sleep Research, 21(1), 10-20.

Laroi, F., \& Woodward, T. S. (2007). Hallucinations from a cognitive perspective. [Review]. Harvard Review of Psychiatry, 15(3), 109-117.

Lerner, A., Rudinski, D., Bor, O., \& Goodman, C. (2013). Flashbacks and HPPD: A clinicaloriented concise review. The Israel journal of Psychiatry and Related Sciences, 51(4), 296-301.

Liikkanen, L. A. (2012). Musical activities predispose to involuntary musical imagery. Psychology of Music, 40(2), 236-256. 
Martínez, J., García, A. S., Oliver, M., Molina, J. P., \& González, P. (2014). VITAKI: A Vibrotactile Prototyping Toolkit for Virtual Reality and Video Games. International Journal of Human-Computer Interaction, 30(11), 855-871.

Mathews, V. P., Kronenberger, W. G., Wang, Y., Lurito, J. T., Lowe, M. J., \& Dunn, D. W, (2005). Media violence exposure and frontal lobe activation measured by functional magnetic resonance imaging in aggressive and nonaggressive adolescents. Journal of Computer Assisted Tomography, 29(3), 287-292.

McGrath, J. J., Saha, S., Al-Hamzawi, A., \& et al. (2015). Psychotic experiences in the general population: A cross-national analysis based on 31261 respondents from 18 countries. JAMA Psychiatry, 72(7), 697-705.

Metcalf, O., \& Pammer, K. (2011). Attentional bias in excessive massively multiplayer online role-playing gamers using a modified Stroop task. Computers in Human Behavior, 27(5), 19421947.

Norman, D. A. (1981). Categorization of action slips. Psychological Review, 88(1), 1-15.

Ohayon, M. M. (2000). Prevalence of hallucinations and their pathological associations in the general population. Psychiatry Research, 97(2-3), 153-164.

Ortiz de Gortari, A. B. (2010). Targeting the Real life Impact of Virtual interactions: The Game Transfer Phenomenon 42 video games players' experiences (Master dissertation). Retrieved from Digitala Vetenskapliga Arkivet database (No. 425850), Stockholm University, Stockholm. 
Ortiz de Gortari, A. B. (2015). Exploring Game Transfer Phenomena: A multimodal research approach for investigating video games' effects (unpublished doctoral dissertation). Nottingham Trent University, Nottingham, UK.

Ortiz de Gortari, A. B., Aronsson, K., \& Griffiths, M. D. (2011). Game Transfer Phenomena in video game playing: A qualitative interview study. International Journal of Cyber Behavior, Psychology and Learning 1(3), 15-33.

Ortiz de Gortari, A. B., Aronsson, K., \& Griffiths, M. D. (2013). Game Transfer Phenomena in video game Playing: A qualitative interview study. In R. Zheng (Ed.), Evolving Psychological and Educational Perspectives on Cyber Behavior (pp. 170-188). Pennsylvania: IGI Global.

Ortiz de Gortari, A. B., \& Griffiths, M. D. (2012). An introduction to Game Transfer Phenomena in video game playing. In J. I. Gackenbach (Ed.), Video Game Play and Consciousness (pp. 223250). Hauppauge, NY: Nova Publisher.

Ortiz de Gortari, A. B., \& Griffiths, M. D. (2014a). Altered visual perception in Game Transfer Phenomena: An empirical self-report study. International Journal of Human-Computer Interaction, 30(2), 95-105.

Ortiz de Gortari, A. B., \& Griffiths, M. D. (2014b). Auditory experiences in Game Transfer Phenomena: An empirical self-report study. International Journal of Cyber Behavior, Psychology and Learning 4(1), 59-75.

Ortiz de Gortari, A. B., \& Griffiths, M. D. (2014c). Automatic mental processes, automatic actions and behaviours in Game Transfer Phenomena: An empirical self-report study using online forum data. International Journal of Mental Health and Addiction, 12(4), 1-21. 
Ortiz de Gortari, A. B., \& Griffiths, M. D. (2015). Game Transfer Phenomena and its associated factors: An exploratory empirical online survey study. Computers in Human Behavior, 51(0), 195-202.

Poels, K., Ijsselsteijn, W. A., \& de Kort, Y. (2014). World of Warcraft, the aftermath: How game elements transfer into perceptions, associations and (day)dreams in the everyday life of massively multiplayer online role-playing game players. New Media and Society, 16, 1-17.

Stickgold, R., Malia, A., Maguire, D., Roddenberry, D., \& O'Connor, M. (2000). Replaying the Game: Hypnagogic images in normals and amnesics. Science, 290(5490), 350-353.

Van Holst, R. J., Lemmens, J. S., Valkenburg, P. M., Peter, J., Veltman, D. J., \& Goudriaan, A. E. (2012). Attentional bias and disinhibition toward gaming cues are related to problem gaming in male adolescents. Journal of Adolescent Health, 50(6), 541-546.

Wamsley, E. J., Perry, K., Djonlagic, I., Reaven, L. B., \& Stickgold, R. (2010). Cognitive replay of visuomotor learning at sleep onset: Temporal dynamics and relationship to task performance. Sleep, 1(33), 59-68.

\section{ABOUT THE AUTHORS}

Dr. Angelica B. Ortiz de Gortari is a researcher in psychology. Her area of expertise is Game Transfer Phenomena (GTP). She has won awards for her research. Her research has featured in variety of media including the TV series CSI: Cyber. She has published academically and presented at several conferences. 
Dr. Mark Griffiths is a Chartered Psychologist and Director of the International Gaming Research Unit at Nottingham Trent University (UK). He has published over 550 refereed research papers, five books, 130+ book chapters and over 1000 other articles, and has won 15 national and international awards for his research. 
Table 1. Socio-demographic characteristics

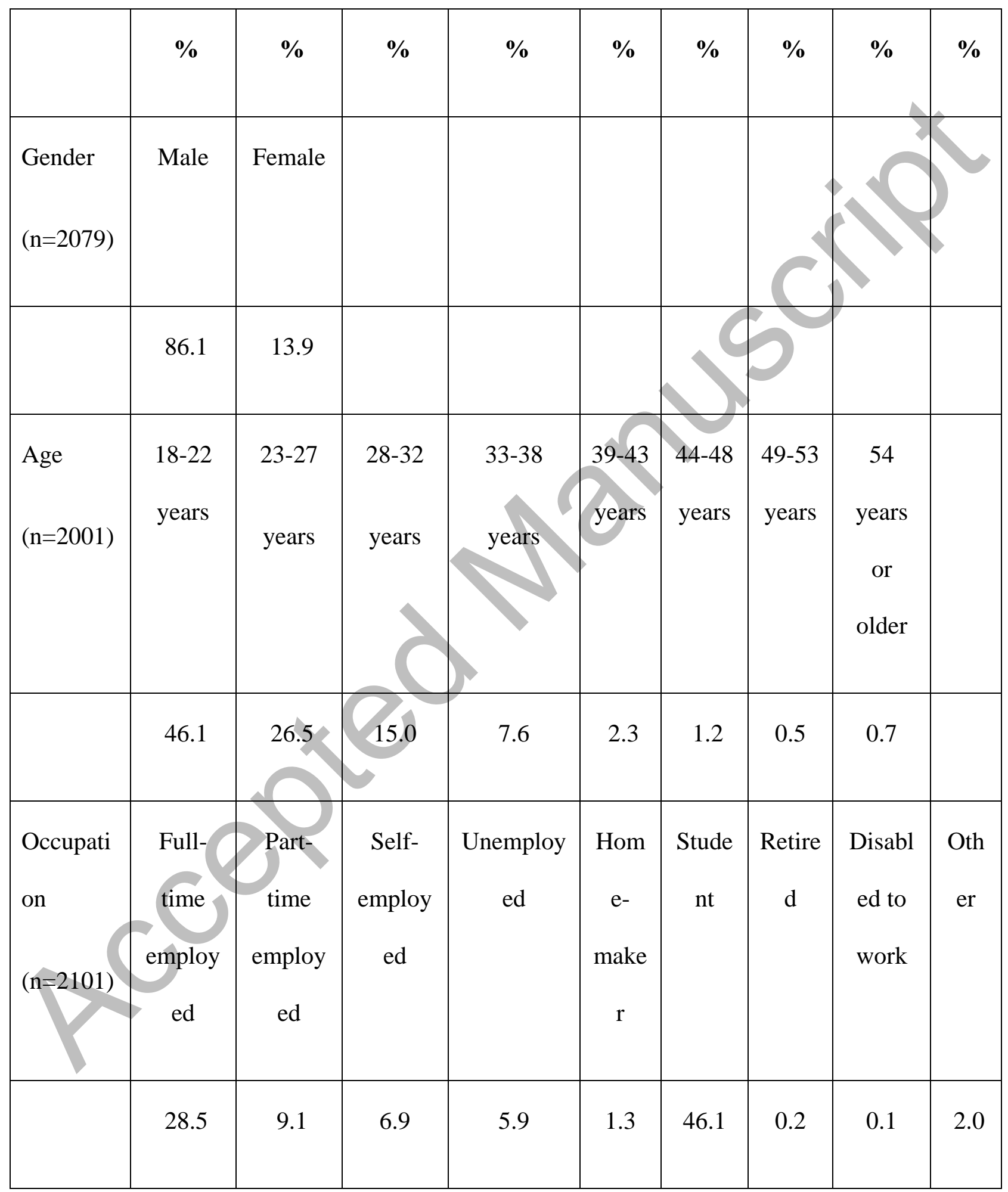


Table 2. Frequencies of gamer profile and gaming habits

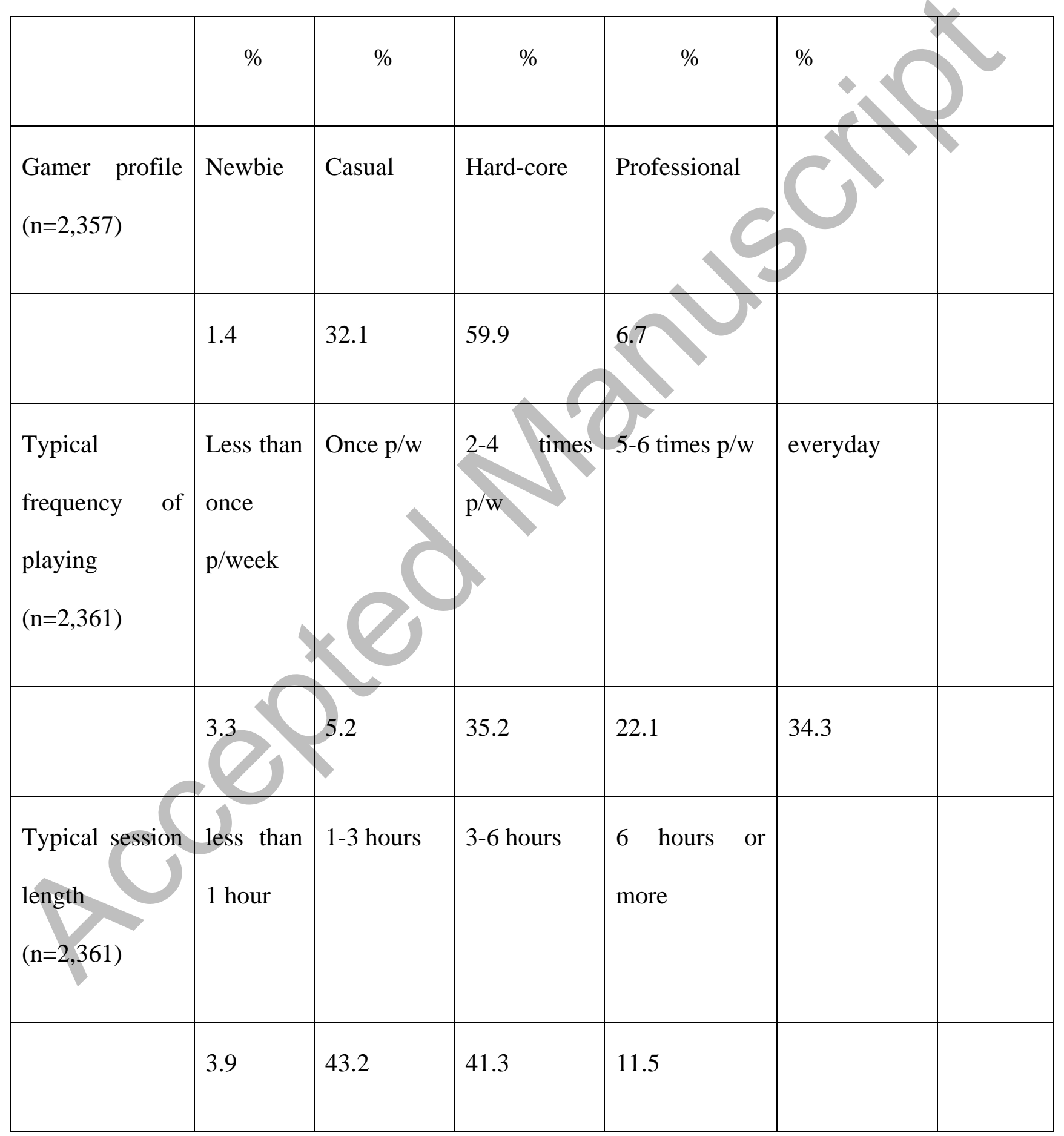




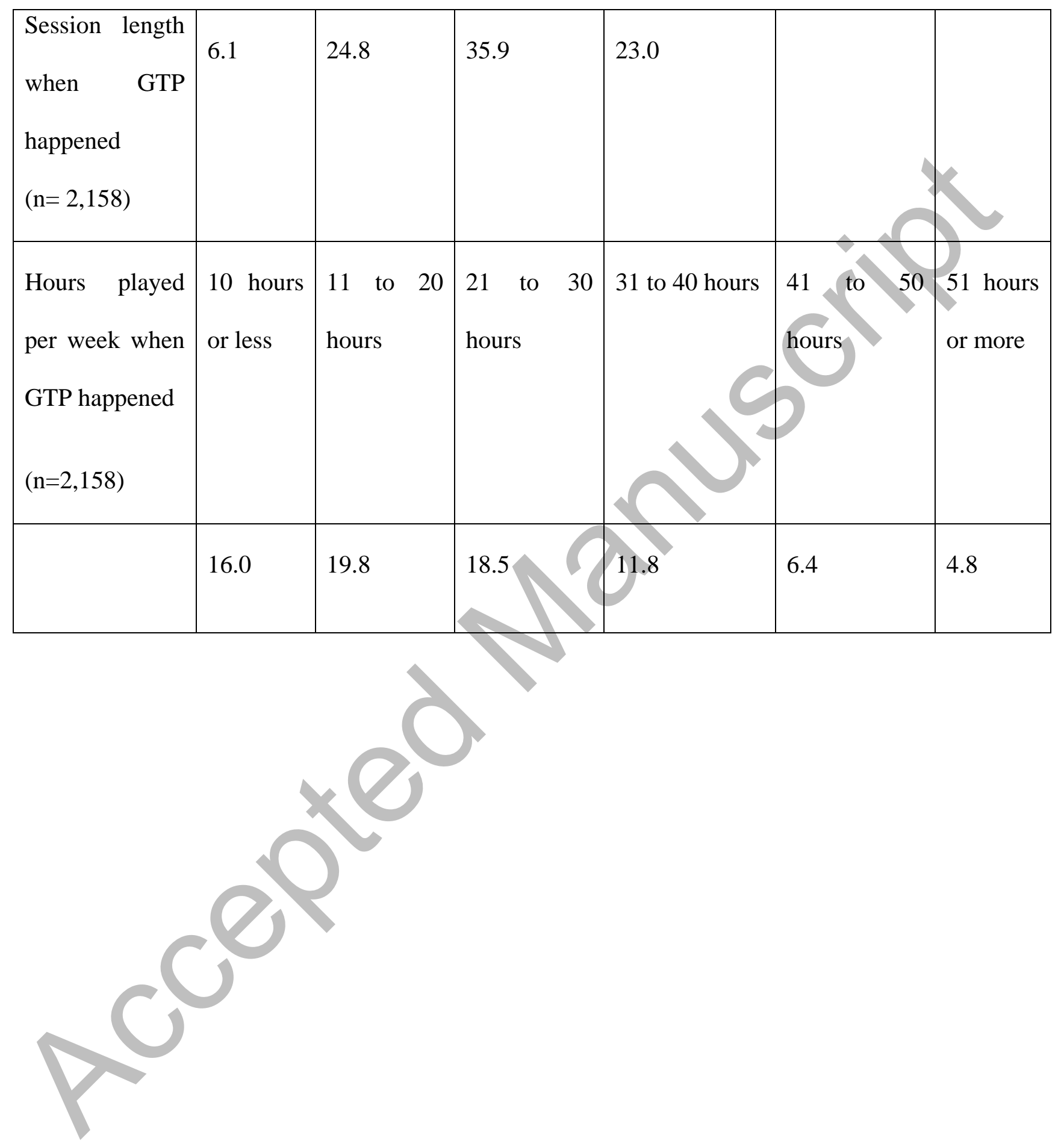


Table 3. Frequencies of GTP modalities, sub-modalities and types $(n=2,362)$

\begin{tabular}{|c|c|}
\hline \multicolumn{2}{|r|}{ Total } \\
\hline I. Altered perceptions & \\
\hline Altered visual perceptions Sub-modality & 84.8 \\
\hline Visualized/seen VG images with closed eyes & 76.8 \\
\hline Misperceived a real life object as something from a VG & 45.5 \\
\hline Seen distorted real life environments and/or objects & 35.7 \\
\hline Seen VG images with open eyes & 30.6 \\
\hline Altered body perceptions Sub-modality & 72.7 \\
\hline Bodily sensations of movement as if being in a VG & 50.9 \\
\hline Perceived time and/or feeling the body differently after playing a VG & 49.3 \\
\hline
\end{tabular}




\begin{tabular}{|l|r|}
\hline Tactile touch sensation associated with a VG & 41.3 \\
\hline Felt as though the mind has disconnected from the body after playing & 28.5 \\
\hline Altered auditory perceptions Sub-modality & 85.2 \\
\hline Heard the music from a VG when not playing & 73.9 \\
\hline Misinterpreted a sound in real life as something from a VG & 65.3 \\
\hline Heard a sound from a VG when not playing & 64.6 \\
\hline Heard a character's voice from a VG when not playing & 74.6 \\
\hline II. Automatic mental processes Modality & 47.9 \\
\hline
\end{tabular}




\begin{tabular}{|l|r|}
\hline Have momentarily mixed up VG events with actual real life events & \\
\hline III. Actions and Behaviors Modality & 42.5 \\
\hline Sang, shouted or said something from a VG in real life unintentionally & \\
\hline Unintentionally acted differently in real life situations because & \\
\hline something experienced in a VG & 47.7 \\
\hline Reflex body reaction associated with a VG & \\
\hline Acted out a behavior or performed an activity influenced by a VG & \\
\hline
\end{tabular}


Table 4. Frequency of characteristics of GTP in terms of videogames associated with GTP, duration, temporal manifestation, circumstances of occurrence and impact on gamers $(n=2,158)$

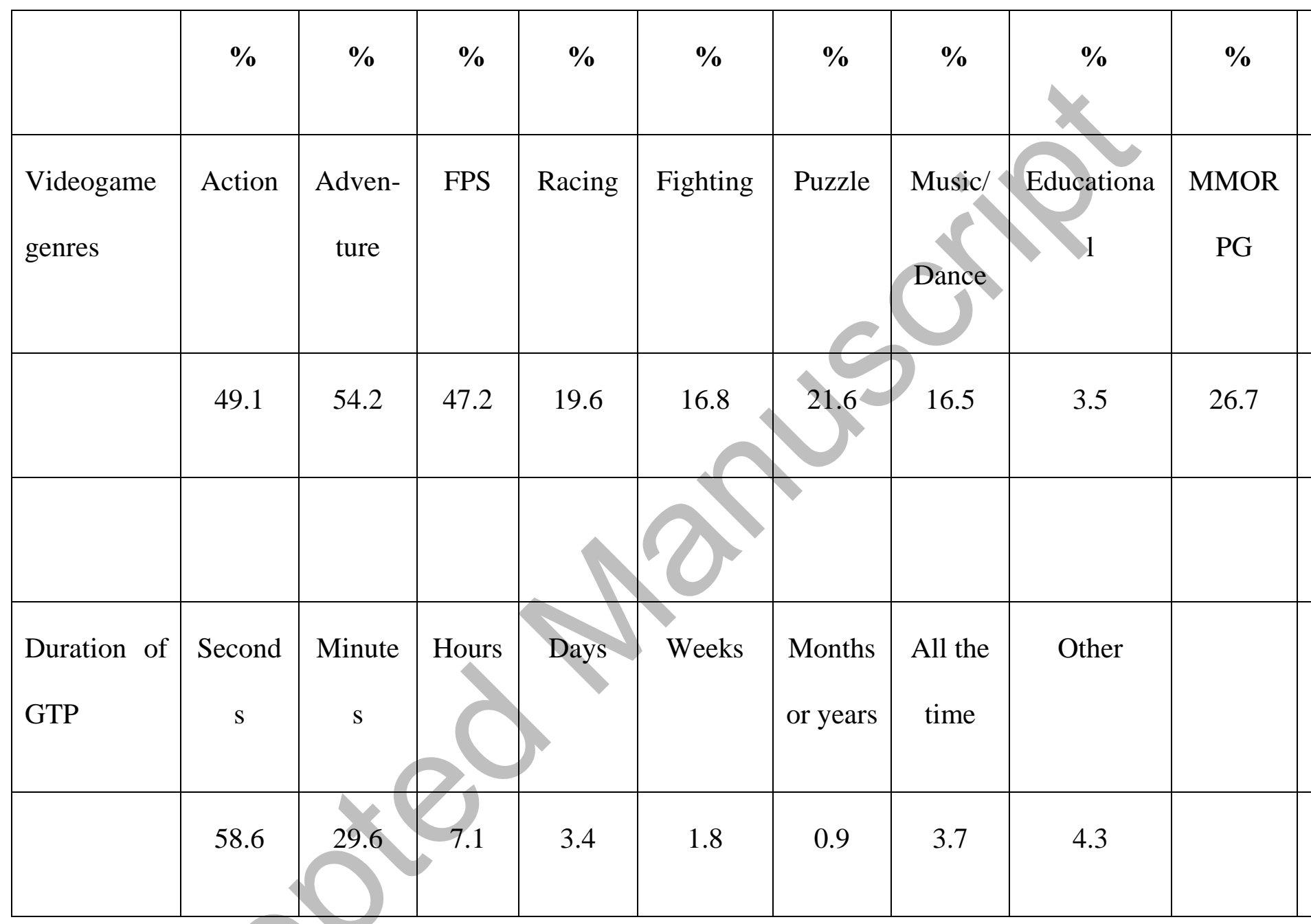

\begin{tabular}{|c|c|c|c|c|c|c|c|}
\hline Manifestation & Experienced & Directly & Hours & Days & Weeks & Other & \\
time of GTP & whilst & after & after & after & or more & & \\
& playing & playing & & & & & \\
& & & & & & & \\
\hline
\end{tabular}




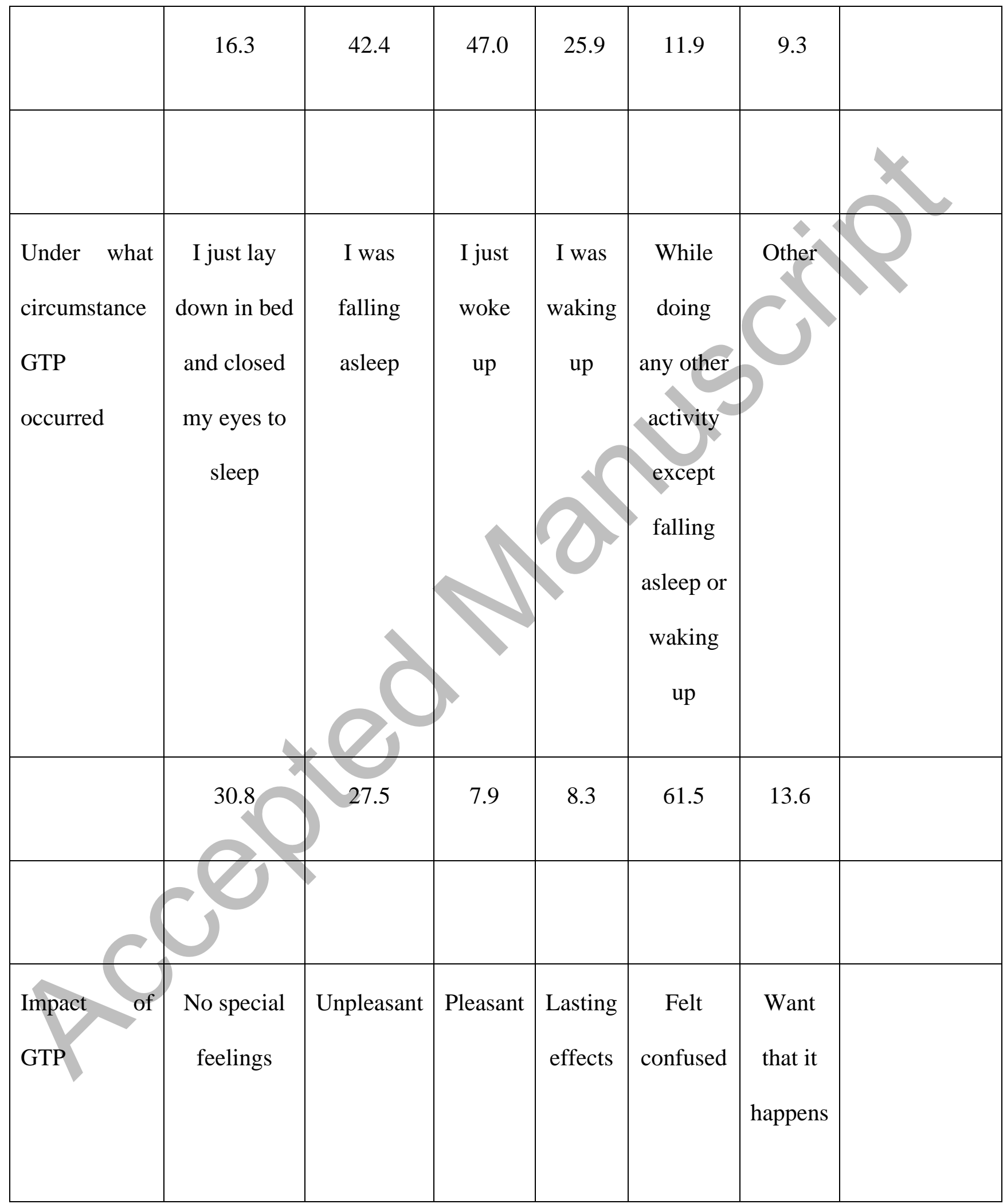




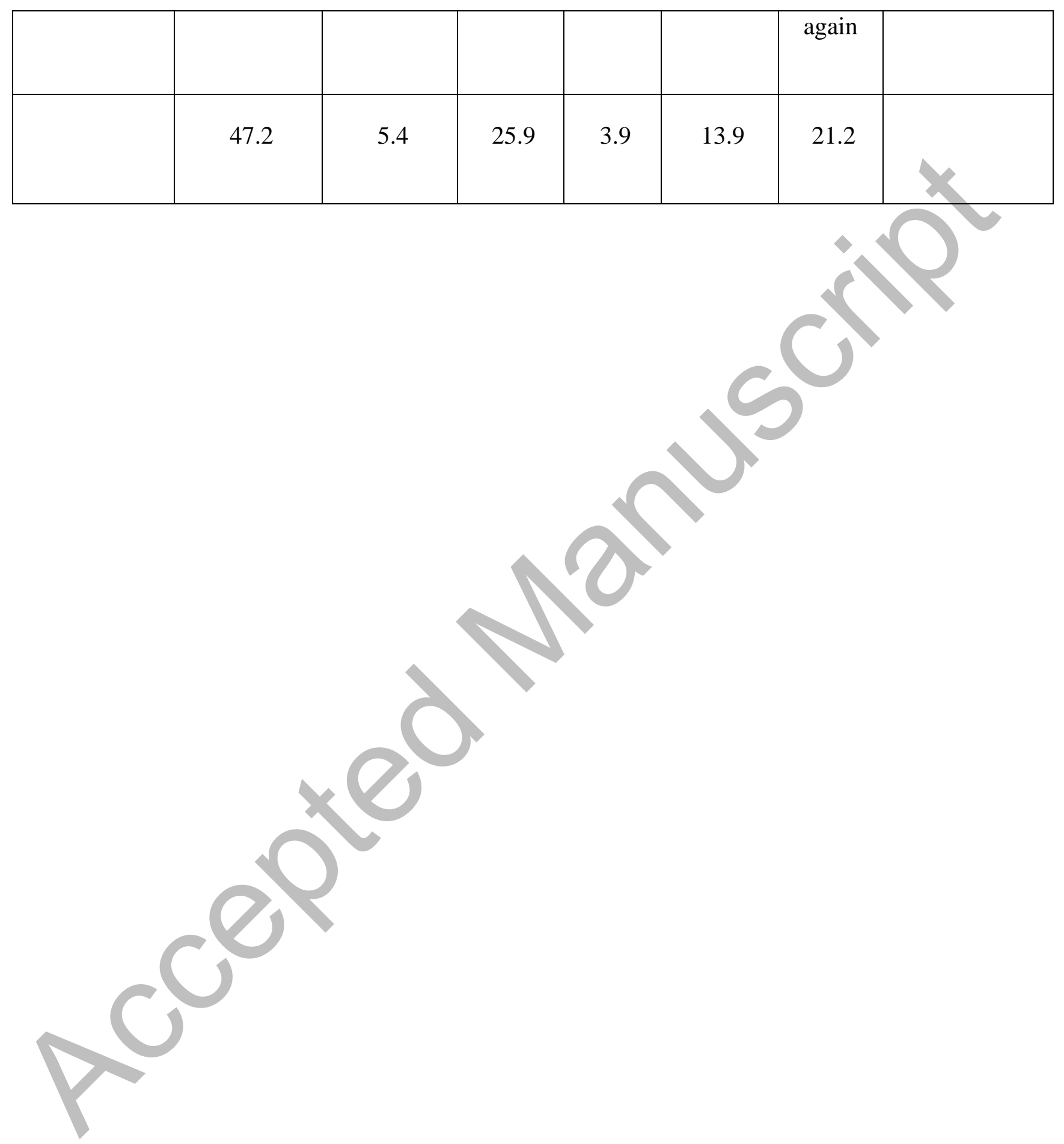

